\title{
Experimental Verification of Torque Sensorless Control for Electric Power-Assisted Bicycles on Sloped Environment
}

\author{
Norihito Fukushima \\ Department of Physics, Electrical and \\ Computer Engineering \\ Yokohama National University \\ fukushima-norihito-sb@ynu.jp
}

\author{
Yasutaka Fujimoto \\ Department of Physics, Electrical and \\ Computer Engineering \\ Yokohama National University \\ fujimoto@ynu.ac.jp
}

\begin{abstract}
Many commercial electric power-assisted bicycles must be equipped an expensive torque sensor to measure the pedaling torque of the user. If it is possible to measure the pedaling torque without such an expensive torque sensor, the cost of these bicycles will be reduced. In this paper, a method for estimating the pedaling torque that is applied in a sloped environment is proposed. To increase the estimation accuracy, the parameter identification of the motor is presented. The validity of the proposed method is shown thorough experiments with motor assistance on a sloped environment.

Index Terms-electric power-assisted bicycle, disturbance observer, Recursive least square method, multiple forgetting factors.
\end{abstract}

\section{INTRODUCTION}

The use of electric power-assisted bicycles is spreading to all generations in many technologically advanced nations because, unlike motorcycles or cars, a special license is not necessary to use them. With this growing popularity of bicycles, many investigations focusing on the control of them have been carried out to increase the convenience of there use. Xuan and Tomizuka proposed a robust disturbance observer based power-assist scheme to provide flexible assistive power even in uphill conditions [1]. Hatada et al. proposed a power assist control method using a periodic disturbance observer that viewed the pedaling torque generated by a human as a periodic disturbance [2]. Their control method achieved a high energy efficiency compared with a general method for commercial electric power-assisted bicycles.

Electric power-assisted bicycles are convenient, but there are multiple laws regarding the output-limiting of motor or the method of motor assistance. In most cases including Japan, the motor output must be driven synchronously with the human pedaling torque, and the range of motor operation is limited by the traveling velocity of the bicycle. Therefore, some sensors such as torque sensors and speed sensors are necessary for bicycles to measure the necessary values for adequate control. Unfortunately, the cost of torque sensors is relatively high, which means that electric power-assisted bicycles to be expensive product. If the human pedaling torque can be estimated without such an expensive torque sensor, the

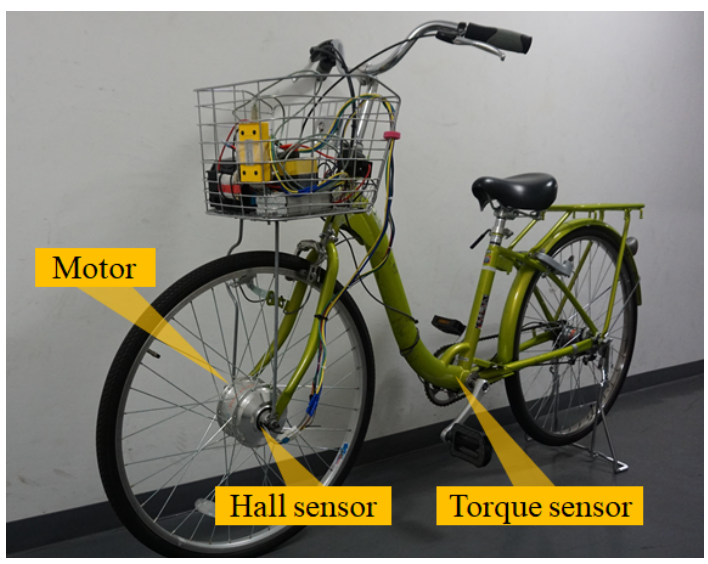

Fig. 1: Experimental bicycle

sensor will no longer be necessary in the bicycle design and it the overall cost will be reduced. This study proposes a method to estimate the pedaling torque without a torque sensor. There have been many studies of torque sensorless control methods to reduce the cost of electric power-assisted bicycles [3]-[5].

In this study, we propose a method to estimate the pedaling torque that focuses on estimating the periodic characteristics of the human pedaling torque with only a hall sensor [6]. We also present an improvement to the recursive least square method to increase the estimation accuracy when operating in sloped environments [7]. The validity of our proposed method is shown in numerical simulations and experiments without motor assistance. The paper details the more accurate model of the disturbance observer using the parameters identified from characteristic of a brushless DC motor. Using this more accurate model, improves the estimation accuracy in the experiments with motor assistance.

The organization of the paper is as follows. The model of our experimental bicycles and the parameter identification are shown in section II, and the method to estimate pedaling torque is shown in III. The experimental condition and results are shown in section IV, and section V contains the conclusions 
of the paper.

\section{MODELING AND IDENTIFICATION}

\section{A. Bicycle longitudinal dynamics}

Fig. 1 shows the experimental bicycle used in this investigation. A brushless DC motor is mounted on the front wheel, and a hall sensor is built inside the motor. The wheel angle and the velocity of the bicycle are obtained from this sensor. The longitudinal dynamics are derived from the bicycle's equation of motion and the equation of motion for the motor built into the front wheel of the bicycle. The physical parameters are defined in Table. I. The bicycle's equation of motion is described as follows:

$$
M \dot{v}=F_{\text {motor }}+F_{\text {man }}-F_{\text {load }}
$$

Equation (1) can be rewritten as the equation of motion for the torque around the axis of the front motor as follows:

$$
J \dot{\omega}=\tau_{\text {motor }}+\tau_{\text {man }}-\tau_{\text {load }}
$$

where $J=M r^{2}, \tau_{\text {motor }}=r F_{\text {motor }}, \tau_{\text {man }}=r F_{\text {man }}$, and $\tau_{\text {load }}=r F_{\text {load }}$, respectively. Assuming that the electrical time constant of the motor is quite short and that the motor is not salient, the q-axis current $I_{q}$ and the motor torque $\tau_{\text {motor }}$ are described by

$$
\begin{aligned}
I_{q} & =\frac{1}{R}\left(V_{q}-K_{e} \omega\right) \\
\tau_{\text {motor }} & =K_{\tau} I_{q}=\frac{K_{\tau}}{R} V_{q}-\frac{K_{\tau} K_{e}}{R} \omega
\end{aligned}
$$

The plant model is obtained from (2) and (4) as follows:

$$
J \dot{\omega}=\frac{K_{\tau}}{R} V_{q}-\frac{K_{\tau} K_{e}}{R} \omega-\tau_{d i s}
$$

where $\tau_{d i s}$ represents the total disturbance torque that we will estimate using a disturbance observer (DOB) [8]. In this study, the disturbance torque is treated as the sum of the load torque and the pedaling torque. Therefore $\tau_{d i s}$ is defined as follows.

$$
\tau_{\text {dis }}=\tau_{\text {load }}-\tau_{\text {man }}
$$

\section{B. Parameter identification of motor}

The Values of the parameters related to the motor torque must be known for an accurate DOB. To identify the necessary parameters, we utilize the linear relationship between torque and angular velocity in a brushless de motor.

The setup for the experimental is shown in Fig. 2. The motor is connected to the torque sensor, there is an incremental rotary encoder, and the powder brake acts as a load. If this experimental setup is regarded as a two-inertia system with a motor and a load side, the equations of this system are as follows:

$$
\begin{aligned}
J_{m} \dot{\omega}_{m} & =\frac{1}{G_{m}}\left(\tau_{\text {motor }}-\tau_{s}\right) \\
J_{l} \dot{\omega} & =\tau_{s}-\tau_{d i s}^{\prime} \\
\omega & =G_{m} \omega_{m}
\end{aligned}
$$

TABLE I: Parameters of electric power-assisted bicycle

\begin{tabular}{c|l}
\hline \hline$V_{q}$ & q-axis voltage of armature windings \\
\hline$I_{q}$ & q-axis current of armature windings \\
\hline$R$ & Resistance of armature windings \\
\hline$K_{e}$ & Constant of back electromotive force \\
\hline$K_{\tau}$ & Torque constant \\
\hline$M$ & Mass of bicycle and rider \\
\hline$J$ & Inertia of front wheel \\
\hline$J_{n}$ & Nominal value of inertia of front wheel \\
\hline$r$ & Wheel radius \\
\hline$\theta_{c}$ & Angle of crank \\
\hline$G_{c}$ & Gear ratio between clank and rear wheel \\
\hline$v$ & Velocity of bicycle \\
\hline$\omega$ & Angular velocity of wheel \\
\hline$F_{\text {motor }}$ & Motor force \\
\hline$F_{\text {man }}$ & Pedaling force \\
\hline$F_{\text {load }}$ & Load force \\
\hline$\tau_{\text {motor }}$ & Motor torque \\
\hline$\tau_{\text {man }}$ & Pedaling torque \\
\hline$\tau_{\text {load }}$ & Load torque \\
\hline \hline
\end{tabular}

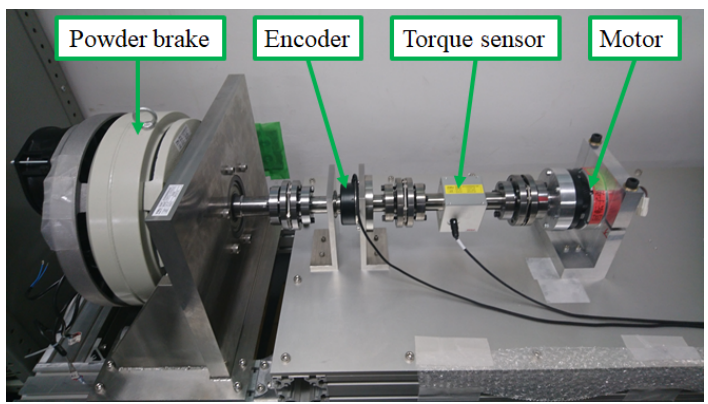

Fig. 2: Experimental setup for identification of motor parameters

where $J_{m}, J_{l}, \omega_{m}, G_{m}, \tau_{s}$ and $\tau_{d i s}^{\prime}$ represent the motorside inertia, the load-side inertia, the motor's angular velocity, the motor's gear ratio, the torsion torque and the load-side disturbance torque, respectively. From (7), (8) and (9), the load-side disturbance torque can be calculated as follows.

$$
\tau_{\text {dis }}^{\prime}=\tau_{\text {motor }}-\left(J_{m} G_{m}{ }^{2}+J_{l}\right) \dot{\omega}
$$

Assuming that the motor rotates at constant speed, the moment of inertia can be neglected in (10). Then, the motor torque matches the load-side disturbance torque measured by the torque sensor.

In the experiment, we applied the duty ratio to command value of the motor. Then we applied the differential load torque multiple times and measured the torque and angular velocity according to the load torque for each duty ratio. The resulting data are shown in Fig. 3. We fit straight lines to each set of data from each duty ratio using least square method. From there results, a linear relationship was obtained between torque and angular velocity in each duty ratio. To take advantage of the linear characteristics of the motor equation, we rewrite (4) as follows:

$$
\tau_{\text {motor }}=X_{1} d+X_{2} \omega+X_{3}
$$




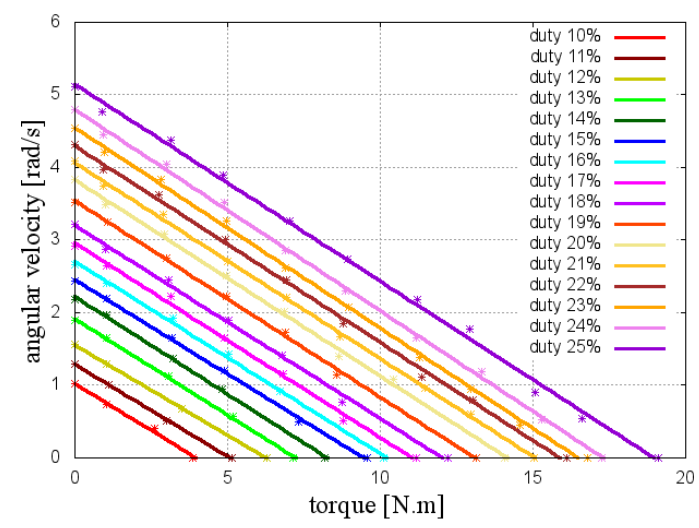

Fig. 3: Linear relationships between load torque and angular velocity

where $d$ is duty ratio, and $X_{1}, X_{2}$ and $X_{3}$ are the equation coefficients. We decided to also consider an offset by adding $X_{3}$ to the equation.

Next, we express equation (11) with its n sets of measurement results in matrix form as follows:

$$
\left[\begin{array}{c}
\tau_{\text {motor } 1} \\
\vdots \\
\tau_{\text {motor } n}
\end{array}\right]=\left[\begin{array}{ccc}
d_{1} & \omega_{1} & 1 \\
\vdots & \vdots & \vdots \\
d_{n} & \omega_{n} & 1
\end{array}\right]\left[\begin{array}{c}
X_{1} \\
X_{2} \\
X_{3}
\end{array}\right]
$$

and which can be rewritten as

$$
\tau=A X
$$

The parameter vector $\boldsymbol{X}$ includes a unknown parameters that can be calculated using least square method as follows.

$$
\boldsymbol{X}=\left(\boldsymbol{A}^{T} \boldsymbol{A}\right)^{-1} \boldsymbol{A}^{T} \boldsymbol{\tau}
$$

The parameters identification results are shown in Table II. Figure. (4) shows the approximated plane of (11) with the calculated $\boldsymbol{X}$ and measurement results. From the equation of the approximated plane, it is apparent that when the case angular velocity is 0 , torque is not generated until the value of $d$ become greater than 5.756. Therefore, there is a nonnegligible dead band between the duty ratio and the torque. In light of this dead band, (11) is rewritten as follows:

$$
\tau_{\text {motor }}=X_{1}\left(d-d_{0}\right)+X_{2} \omega
$$

where $d_{0}=5.756$. Using calculated $X_{1}$ and $X_{2}$, the plant model is expressed as follows.

$$
\begin{cases}J \dot{\omega}=X_{1}\left(d-d_{0}\right)+X_{2} \omega-\tau_{d i s} & \left(d>d_{0}\right) \\ J \dot{\omega}=X_{2} \omega-\tau_{d i s} & \left(d \leq d_{0}\right)\end{cases}
$$

\section{Method FOR PEDALING TORQue ESTIMATION}

This section describes the method to estimate the pedaling torque. From (6), the estimated pedaling torque is calculated by

$$
\hat{\tau}_{\text {man }}(t)=\hat{\tau}_{\text {load }}(t)-\hat{\tau}_{\text {dis }}(t)
$$

TABLE II: Identification result

\begin{tabular}{lc}
\hline \hline Parameter & value \\
\hline$X_{1}[\mathrm{Nm}]$ & 0.976 \\
\hline$X_{2}[\mathrm{Nms} / \mathrm{rad}]$ & -3.678 \\
\hline$X_{3}[\mathrm{Nm}]$ & -5.616 \\
\hline \hline
\end{tabular}

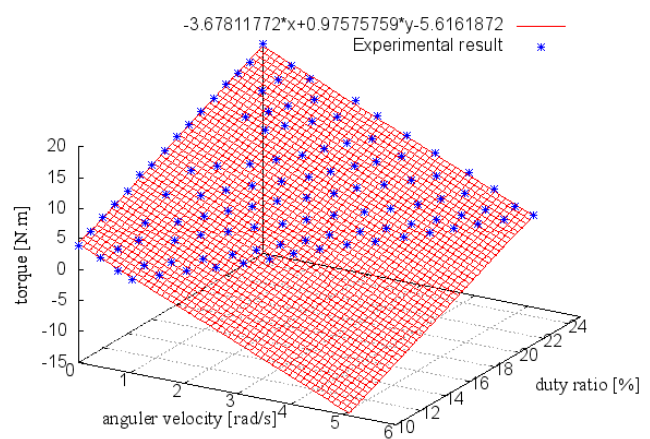

Fig. 4: Approximated plane of measured torque

where $\hat{\tau}_{\text {load }}(t), \hat{\tau}_{\text {dis }}(t)$ are the estimated load torque and estimated disturbance torque, respectively. If the load torque and the disturbance torque are estimated accurately, the pedaling torque can also be estimated.

\section{A. Disturbance Obserber}

We first introduce a DOB to estimate the disturbance torque. From the rewritten plant model (16), a DOB is constructed as follows:

$$
\begin{cases}\hat{\tau}_{d i s}(s)=\frac{\omega_{d}{ }^{2}}{s^{2}+\sqrt{2} \omega_{d} s+\omega_{d}{ }^{2}}\left(X_{1}\left(d-d_{0}\right)+X_{2} \omega-J_{n} s \omega\right) \\ \hat{\tau}_{d i s}(s)=\frac{\omega_{d}{ }^{2}}{s^{2}+\sqrt{2} \omega_{d} s+\omega_{d}{ }^{2}}\left(X_{2} \omega-J_{n} s \omega\right) & \text { if } \quad\left(d>d_{0}\right) \\ & \text { if } \quad\left(d \leq d_{0}\right)\end{cases}
$$

where $\omega_{d}$ is the cut-off frequency of the DOB. In our experimental unit, the motor is equipped with hall sensors to measure electric angle of the rotor. Therefore, we calculate the angular velocity of the bicycle using hall sensors. In addition, we utilize a second-order low pass filter in the DOB to suppress the observation noise.

\section{B. Estimation of Load Torque}

Next, we describe the estimation method for the load torque. Assuming that the load torque does not change suddenly while the bicycle is in motion, we first estimate the average load torque $\tilde{\tau}_{\text {load }}\left(\theta_{c}\right)$ by using the average pedaling torque $\tilde{\tau}_{\text {man }}\left(\theta_{c}\right)$ and the average disturbance torque $\tilde{\tau}_{\text {dis }}\left(\theta_{c}\right)$ as follows:

$$
\tilde{\tau}_{\text {load }}\left(\theta_{c}\right)=\tilde{\tau}_{\text {dis }}\left(\theta_{c}\right)+\tilde{\tau}_{\text {man }}\left(\theta_{c}\right)
$$


where, $\theta_{c}$ is the crank angle which is obtained from the wheel angle $\theta_{\text {wheel }}$ as follows:

$$
\theta_{c}=G_{c} \theta_{\text {wheel }}
$$

where $G_{c}$ is the gear ratio between the crank and the sprocket of the rear wheel connected by the chain.

Note that these values are the average, not the instantaneous. To estimate the instantaneous load torque, we utilize the recursive least square (RLS) method. This subsection is organized as follows. The method to estimate the average load torque is described in section III-B1. Then, the model of load torque with the RLS method is shown in section III-B2. Finally, the algorithm of the RLS method is described in section III-B3.

1) Estimation of average load torque: The average disturbance torque is approximated as follows.

$$
\tilde{\tau}_{d i s}\left(\theta_{c}(t)\right)=\hat{\tau}_{\text {dis }}(t)
$$

Then, we focus on the periodic characteristic of the pedaling motion with respect to crank angle in order to estimate the average pedaling torque. By applying a Fourier series expansion to the disturbance torque, which includes the pedaling torque, we can reconstruct the components of the pedaling torque as follows:

$$
\begin{aligned}
a_{i} & =\frac{1}{\pi} \int_{0}^{2 \pi} \tilde{\tau}_{d i s}\left(\theta_{c}\right) \cos i \theta_{c} d \theta_{c} \\
b_{i} & =\frac{1}{\pi} \int_{0}^{2 \pi} \tilde{\tau}_{d i s}\left(\theta_{c}\right) \sin i \theta_{c} d \theta_{c}
\end{aligned}
$$

Using the above components, the average pedaling torque in the preceding cycle can be reconstructed as follows:

$$
\tilde{\tau}_{\text {man }}\left(\theta_{c}\right)=\sum_{i=1}^{2}\left(a_{i} \cos i \theta_{c}+b_{i} \sin i \theta_{c}\right)-C
$$

where, term $C$ is the local minimum value during a crank period. The subtraction of $C$ is required to satisfy $\tilde{\tau}_{\text {man }}\left(\theta_{c}\right) \geq$ 0 because the sum of sine and cosine wave result in an average value of 0 . The local minimum value is obtained via the Ferrari formula with a differential value of (24) by $\theta_{c}$.

2) Model of load torque: In this study, we assume that the load torque includes the rolling torque, the torque due to the inclined road, and the aerodynamic drag torque. The load torque is therefore expressed as follows:

$$
\tau_{\text {load }}=\mu M g r+M g r \sin \delta+\frac{1}{2} \rho A C_{d} r^{3} \omega^{2}
$$

The parameters in the equation are shown in Table III. The values of $\mu$ and $C_{d}$ are determined from references [9] and [10], and the numerical value of $\delta$ is evaluated assuming a 7\% upward slope. This equation can be divided into a terms that is proportional to the known parameter $M g r$ and a terms that is proportional to the square of the angular velocity as follows:

$$
\tau_{\text {load }}=M g r K_{1}+K_{2} \omega^{2}
$$

where $K_{1}=\mu+\sin \delta$ and $K_{2}=\frac{1}{2} \rho A C_{d} r^{3}$. From (26), the unknown parameters $K_{1}$ and $K_{2}$ can be estimated in real time
TABLE III: Parameter of load torque

\begin{tabular}{llc}
\hline \hline$\mu$ & Coefficient of rolling friction resistance & 0.007 \\
\hline$g\left[\mathrm{~m} / \mathrm{s}^{2}\right]$ & Acceleration of gravity & 9.8 \\
\hline$\delta[$ degree $]$ & Angle of inclination & 0.07 \\
\hline$\rho\left[\mathrm{Ns}^{2} / \mathrm{m}^{4}\right]$ & Atmospheric density & 1.205 \\
\hline$A\left[\mathrm{~m}^{2}\right]$ & Frontal projected area & 0.72 \\
\hline$C_{d}$ & Air resistance coefficient & 0.9 \\
\hline \hline
\end{tabular}

via RLS method if $\tau_{\text {load }}, M g r$ and $\omega$ are obtained. In this study, we substitute $\tilde{\tau}_{\text {load }}\left(\theta_{c}\right)$ for $\tau_{\text {load }}$ in order to apply the RLS method. The resulting relationship for the linear model is expressed in (27) to (30).

$$
\begin{aligned}
y & =\boldsymbol{\phi}^{T} \boldsymbol{\theta} \\
\boldsymbol{\theta} & =\left[\begin{array}{ll}
K_{1} & K_{2}
\end{array}\right]^{T} \\
\boldsymbol{\phi} & =\left[\begin{array}{ll}
M g r & \omega^{2}
\end{array}\right]^{T} \\
y & =\tilde{\tau}_{\text {load }}\left(\theta_{c}\right)
\end{aligned}
$$

3) RLS method with multiple forgetting factors: The RLS method with a single forgetting factor has been previously proposed for a time-varying parameter estimation [11]. However, this method has a disadvantage in that it is not effective in system with both time-varying parameters and nearly time-constant parameters because the method fundamentally assumes that the parameters vary with similar rate [12]. In this study, it is a possible that the value of $K_{1}$ will change rapidly, perhaps due to a slope, but the value of $K_{2}$ will be nearly constant since aerodynamic drag values are the almost same during travel. Therefore, we introduce a RLS method with multiple forgetting factors to estimate a constant parameter and a time-varying parameter at the same time [12]. The algorithm for this proposed method is expressed as follows:

$$
\hat{\boldsymbol{\theta}}(k)=\hat{\boldsymbol{\theta}}(k-1)+\boldsymbol{L}^{\prime}(k)\left(y(k)-\boldsymbol{\phi}^{T}(k) \boldsymbol{\theta}(k-1)\right)
$$

$\boldsymbol{L}^{\prime}(k)=\frac{1}{1+\frac{P_{1}(k-1) \phi_{1}(k)^{2}}{\lambda_{1}}+\frac{P_{2}(k-1) \phi_{2}(k)^{2}}{\lambda_{2}}}\left[\begin{array}{l}\frac{P_{1}(k-1) \phi_{1}(k)}{\lambda_{1}} \\ \frac{P_{2}(k-1) \phi_{2}(k)}{\lambda_{2}}\end{array}\right]$

$L_{1}(k)=\frac{P_{1}(k-1) \phi_{1}(k)}{\lambda_{1}+P_{1}(k-1) \phi_{1}(k)^{2}}$

$P_{1}(k)=\frac{1}{\lambda_{1}}\left(1-L_{1}(k) \phi_{1}(k)\right) P_{1}(k-1)$

$L_{2}(k)=\frac{P_{2}(k-1) \phi_{2}(k)}{\lambda_{2}+P_{2}(k-1) \phi_{2}(k)^{2}}$

$P_{2}(k)=\frac{1}{\lambda_{2}}\left(1-L_{2}(k) \phi_{2}(k)\right) P_{2}(k-1)$

where $\boldsymbol{L}^{\prime}(k), L_{1}(k), L_{2}(k), P_{1}(k), P_{2}(k)$ are the update gain vector, the update gain of $K_{1}$, the update gain of $K_{2}$, the covariance of $K_{1}$, and the covariance of $K_{2}$ respectively. The variables corresponding to $K_{1}$ and $K_{2}$ are denoted by subscripts 1 and 2, respectively.

Unlike the RLS method with a single forgetting factor, the RLS method with multiple forgetting factors does not have a covariance matrix. Instead, the parameters are updated 


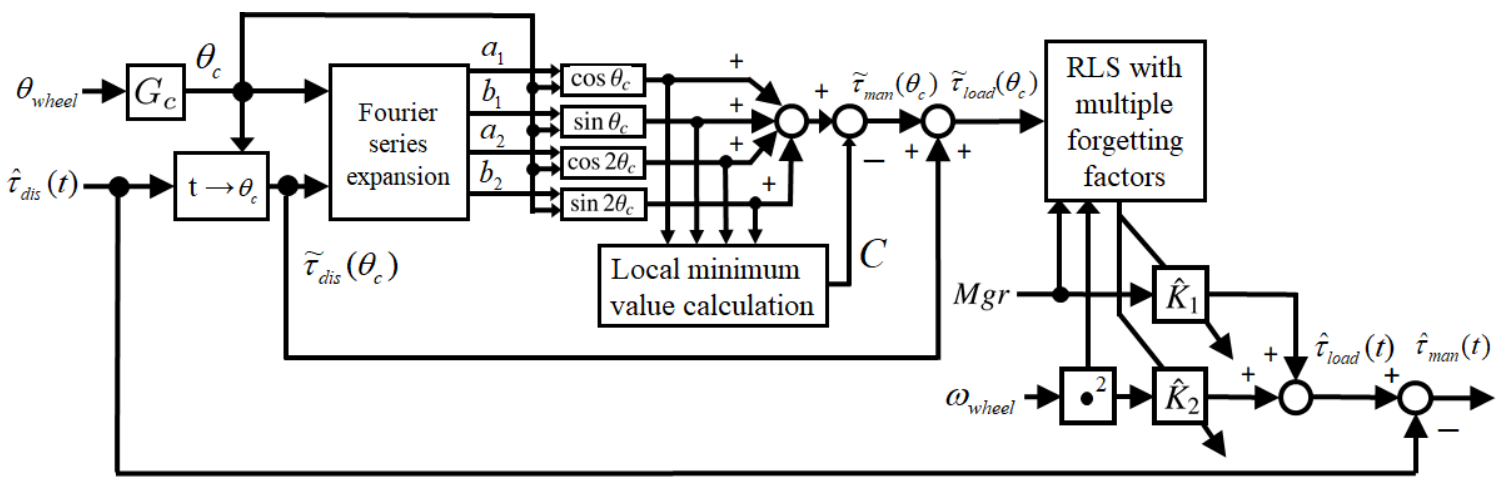

Fig. 5: The block diagram of the estimation of the pedaling torque

according to each forgetting factor, thereby decouples the update step from the covariance of different parameters.

Fig. 5 shows the block diagram of the method for the estimation of the pedaling torque.

\section{Estimation during the initial crank cycle}

During the initial clank cycle, there is an error in the average pedaling torque because there is no data from the preceding cycle. This error in the average pedaling torque results in estimation error in the load torque. To address this issue, the two methods can be applied. The first method is to add an additional update condition to the RLS method. With this method, however, because the value of $\tilde{\tau}_{\text {load }}\left(\theta_{c}\right)$ is estimated from the average pedaling torque, the estimation accuracy of the RLS method is decreased. Therefore, the RLS method is not applied in the initial crank cycle and $K_{1}$ and $K_{2}$ are held at the initial values.

The second possible method is the estimation method for the pedaling torque. As discussed above, the accuracy of the estimated pedaling torque is low because the load toque is constant. To increase the accuracy of the estimated pedaling torque, we assume the load torque is negligibly small during the initial crank cycle, and we use the disturbance torque as the estimated pedaling torque as follows.

$$
\hat{\tau}_{\text {man }}(t)=-\hat{\tau}_{\text {dis }}(t)
$$

\section{EXPERIMENTS}

This section contains the experimental results with motor assistance.

\section{A. Experimental condition}

In this experiment, the estimated pedaling torque was used as the command value to the motor. A torque sensor located in the crank box of the bicycle was used to compare the measured and estimated pedaling torques. The experimental parameters are shown in Table IV. The experiment was conducted on a flat paved road that features an approximately $7 \%$ upward slope at one end. The experiment begun on the flat road and proceeded up the upward slope.
TABLE IV: Experimental parameters

\begin{tabular}{llc}
\hline \hline$J_{n}\left[\mathrm{kgm} m^{2}\right]$ & Inertia of front wheel & $8.49(M=78[\mathrm{~kg}], r=0.33[\mathrm{~m}])$ \\
\hline$\omega_{d}[\mathrm{rad} / \mathrm{s}]$ & Cut-off frequency of DOB & 25 \\
\hline$\lambda_{1}$ & Forgetting factor for $K_{1}$ & 0.997 \\
\hline$\lambda_{2}$ & Forgetting factor for $K_{2}$ & 0.9997 \\
\hline \hline
\end{tabular}

\section{B. Experimental results}

The experimental results for the bicycle traveling on the uphill slope are shown in Fig. 6. In the figure, the red and blue lines represent the actual value from the sensor and the estimated value, respectively. Fig. 6 (a) shows the angular velocity of wheel. Fig. 6 (b) and Fig. 6 (c) show the estimated load torque coefficients $K_{1}$ and $K_{2}$, respectively. The fluctuations in the estimated values were large during the initial estimation step, but the values eventually converged to an approximately constant value. Theoretical values of $K_{1}$ and $K_{2}$ are approximately 0.077 and 0.014 from the Table III. From Fig. 6 (b), we can confirm that the value of estimated $K_{1}$ became large from the initial value due to change of slope. From Fig. 6 (c), on the other hand, the value of estimated $K_{2}$ is not much changed from the initial value even when the bicycle is traveling on the slope. Therefore, we can conclude that the validity of the RLS method with multiple forgetting factors has been confirmed. However, these estimated values were converged to the value larger than the theoretical values. This is due to some estimation error of the the average pedaling torque shown in Fig. 6 (d). Fig. 6 (e) shows the estimated pedaling torque and motor torque, which are represented with the blue and the green lines, respectively. The pedaling torque was estimated fairly accurately, but is apparent that some of the values were over-estimated. The reason of the error is over-estimation of the load torque coefficients $K_{1}$ and $K_{2}$. Moreover, these over-estimated values occurred when the motor torque fluctuation was large. It is therefore assumed that the DOB was not accurate in this situation. Future works will focus on increasing the accuracy of the DOB when the disturbance fluctuation are large by confirming the tracking performance and accuracy of the angular velocity. 


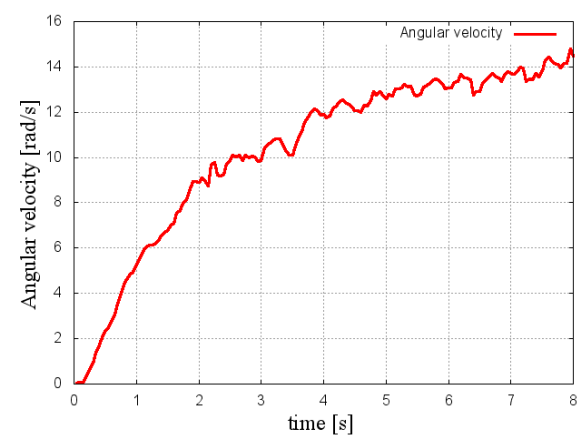

(a) Angular velocity

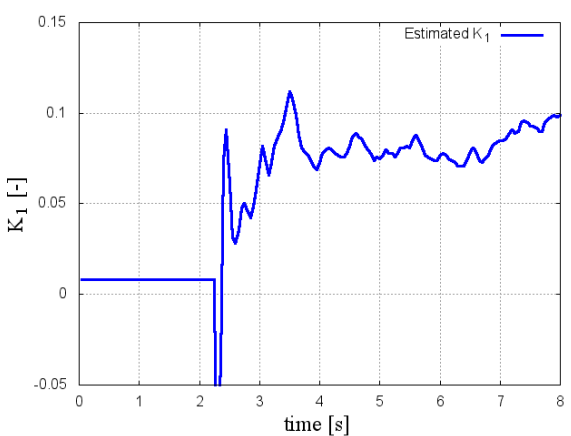

(b) Estimated $K_{1}$

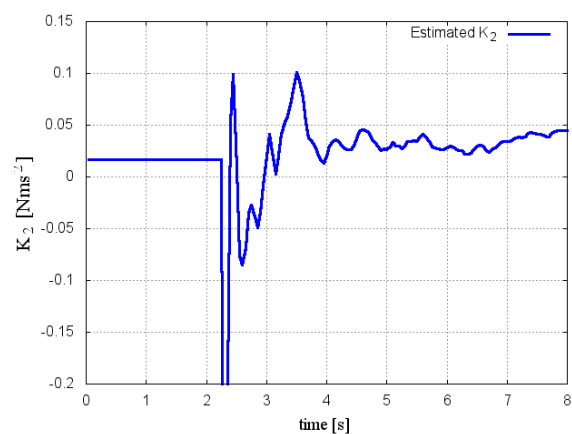

(c) Estimated $K_{2}$

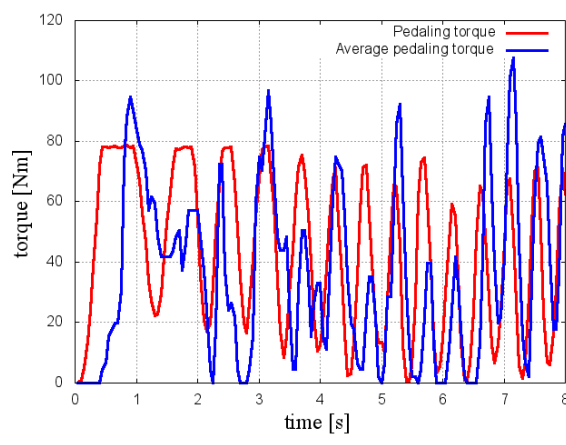

(d) Average pedaling torque

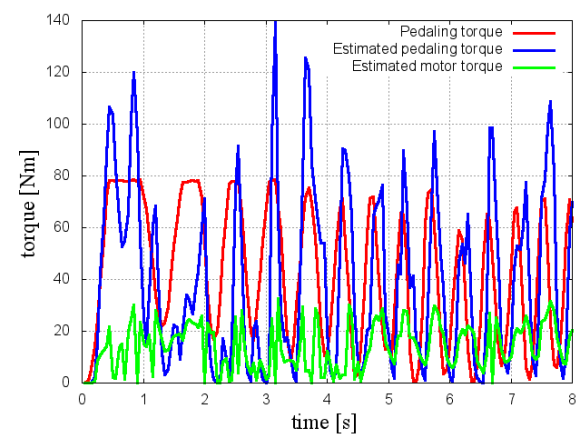

(e) Estimated pedaling torque

Fig. 6: Experimental results

\section{CONCLUSION}

In this paper, the validity of the method for estimating pedaling torque using multiple forgetting factors was experimentally investigated. By modeling the DOB using identified motor parameters and RLS method, the load torque was approximately estimated. As a result, we confirmed that the motorassisted pedaling torque in uphill conditions was estimated with a sufficient degree of accuracy. Future work will focus the improving of estimation accuracy when the the change in the disturbance is large.

\section{ACKNOWLEDGMENT}

We would like to thank Takeji Tokumaru, Electric-bike Corporation Co. Ltd for providing the experimental units.

\section{REFERENCES}

[1] F. Xuan and M. Tomizuka, "Robust disturbance observer design for a power-assist electric bicycle," Proc. of the American Control Conference, pp. 1166-1171, 2010.

[2] K. Hatada, K. Hirata and T. Sato "Energy-efficient power assist control with periodic disturbance observer and its experimental verication using an electric bicycle," SICE Journal of Control, Measurement, and System Integration, vol. 10, no. 5, pp. 410-417, 2017.

[3] V. Sankaranarayanan and S. Ravichandran, "Torque sensorless control of a human-electric hybrid bicycle," Proc. Int. Conf. on Industrial Instrumentation and Control, pp. 806-810, 2015.

[4] DS. Cheon and KH. Nam, "Pedaling torque sensor-less power assist control of an electric bike via model-based impedance control," International Journal of Automotive Technology, vol. 18, no. 2, pp. 327-333, 2017.

[5] H. Kawajiri, H. Mizoguchi, S. Sakaino and T. Tsuji, "Sensorless Pedaling Torque Estimation by Front and Rear Wheels Independently Driven Power Assist Bicycle," Proc. IEEE ICM, pp. 360-365, 2015.
[6] T. Kurosawa and Y. Fujimoto, "Torque Sensorless Control for Electric Power Assisted Bicycle with Instantaneous Pedaling Torque Estimation," IEEJ Jornal of Industry Apprications, vol. 6, no. 2, pp.124-129, 2016.

[7] N. Fukushima and Y. Fujimoto, "Estimation of pedaling torque for electric power-assisted bicycle on slope environment," Proc. IEEE AIM, pp. 1682-1687, 2017.

[8] K. Ohnishi, M. Shibata, and T. Murakami, "Robust Motion Control for advanced mechatronics," IEEE/ASME Transaction on Mechatronics, vol. 1, no. 1, pp.56-67, 1996.

[9] C. Hölzel and F. Höchtl, "Cycling comfort on different road surfaces," Procedia Engineering, vol. 34, pp. 479-484, 2012.

[10] F. R. Whitt and D. G. Wilson, Bicycling science, MIT Press, 1982.

[11] K. J. Astrom and B. Wittenmark, Adaptive Control, Dover Publications, 2008.

[12] A. Vahidi, A. Stefanopoulou, and H. Peng, "Recursive least squares with forgetting for online estimation of vehicle mass and road grade: theory and experiments," Vehicle System Dynamics, vol. 43, no. 1, pp. 31-55, 2005. 